\title{
Peripheral Airway Responsiveness to Sub-maximal Exercise in Asymptomatic Cigarette Smokers.
}

\author{
Pokhrel BR, ${ }^{1}$ Chatopadhyaya $\mathrm{S},{ }^{2}$ Paudel BH. ${ }^{3}$
}

\author{
${ }^{1}$ Department of Physiology \\ Kathmandu Univerisity School of Medical Sciences \\ Dhulikhel, Nepal
}

${ }^{2}$ Department of Physiology

Kalinga Institute of Medical Sciences

Orrisa, India

${ }^{3}$ Department of Physiology

BPKIHS

Dharan, Nepal.

\section{Corresponding Author}

Bhola R Pokhrel

Department of Physiology

Kathmandu Univerisity School of Medical Sciences

Dhulikhel, Nepal

Email:

\section{Citation}

Pokhrel BR, Chatopadhyaya S, Paudel BH. Peripheral airway responsiveness to submaximalexercise in asymptomatic cigarette smokers. Kathmandu Univ Med J 2011;35(3):159-64.

\section{ABSTRACT \\ Background}

Cigarette smoking is one of the cardinal causes for the development of bronchial hyperresponsiveness among the smokers.

\section{Objectives}

This study was perspectively designed to determine the peripheral bronchial responsiveness to sub-maximal exercise challenge in the asymptomatic smokers.

\begin{abstract}
Methods
The subjects were between age of 18-25 years without any findings of cardiorespiratory diseases. We performed the $5 \mathrm{~min}$ step test exercise at intensity of 80 to $90 \%$ of maximum predicted heart rate in 42 young adult male asymptomatic smokers to examine the effect of cigarette smoking on airway responsiveness. Forced expiratory spirogram was recorded before and at 0, 5, 10, $15 \mathrm{~min}$ after the completion of exercise. Pre- to post exercise drop in Forced Expiratory Volume in first second $\geq 15 \%$ was considered hyperresponsive to the challenge.
\end{abstract}

\section{Result}

The analysis of data (mean \pm SE) indicated the bronchial hyper-responsiveness in $22(52 \%)$ smokers. The post exercise recovery time pattern showed drop in forced expiratory spirogram from the resting baseline in the responsive smokers and the maximum percentage fall in the parameters or increase in airway resistance which reflect the peripheral airway integrity such as Forced Expiratory Flow 25\% (20.30 \pm 2.18 Vs $7.88 \pm 3.23, p<0.01$ ), Forced Expiratory Flow $50 \%$ (18.46 \pm 4.40 Vs $1.93 \pm 2.78$, $\mathrm{p}<0.01)$, Forced Expiratory Flow $75 \%(23.94 \pm 3.68$ Vs $0.80 \pm 4.72, \mathrm{p}<0.001)$ and Forced Expiratory Flow $25-75 \%(32.50 \pm 4.79$ Vs $3.64 \pm 3.32, p<0.001)$ was significantly higher in the responsive than non-responsive subgroup of the smokers.

\section{Conclusion}

The occurrence of peripheral airway resistance is more in the responsive than nonresponsive subset of smokers to the exercise challenge and hence more prone to develop obstructive airway disease in the long run.

\section{KEY WORDS}

airway hyper-responsiveness, cigarette smokers, forced expiratory flow.

\section{INTRODUCTION}

Development of bronchial hyperresponsiveness due to active or passive smoking has been found to be linked with the development of several obstructive airway diseases such as chronic obstructive pulmonary disease. ${ }^{1-3}$ The small airway obstruction as shown by several small airway tests is the first stage in protracted process eventually leading to chronic airway flow obstruction..$^{1-3}$ Studies have also revealed that the commencement of bronchial hyper responsiveness most often correlates pathologically with the development of airway diseases, primarily in the peripheral airways. ${ }^{4-7}$ So, the assessment of the cigarette smoke-induced airway changes through the intervention of challenges such as histamine, methacholine, including exercise is an important approximation of respiratory system, especially for bronchial responsiveness and airway resistance. To serve the purpose, the asymptomatic smokers have been taken as a model of study to record the smoke induced earlier change in airway tract.

There are limited studies on asymptomatic smokers to find the degree of bronchial hyper-responsiveness (BHR) 
which is linked to several obstructive diseases, rarely reporting on peripheral airways, and most of them are based on pharmacological challenge. Exercise as a nonpharmacological and non-invasive tool, we employed among age, height, and weight matched asymptomatic young adult smokers to find bronchial responsiveness to submaximal exercise challenge. Post exercise maximum fall in (Forced Expiratory Volume in first second; FEV1) more than $15 \%$ has been considered as the abnormal bronchial response to exercise in asymptomatic smokers. Further in the study, analysis of data has been done with an objective to measure the peripheral airway responsiveness in both responsive and non-responsive subgroup of smokers.

\section{METHODS}

\section{Subjects}

The study was conducted at BP Koirala Institute of Health Sciences, Dharan in 2006 and completed at Nepalgunj Medical College in 2009. In this retrospective study, we recruited forty-two male tobacco smokers aged between 18 to 25 years from Nepalgunj Medical College (NGMC), Chisapani, Banke, Nepal and BP Koirala Institute of Health Sciences (BPKIHS), Dharan, Nepal, by simple random sampling technique. They consisted of volunteers attending medical conference, hospital workers, or medical students. A questionnaire was used to collect the following information from each subject: personal data, age, sex, place of growing, and occupation, and all were screened by physician-acquired history. Subjects were excluded from the study if there was any exercise discomfort and history of wheezing, taking any medications that might influence airway tone, current cough, dyspnoea, sputum production, asthma, allergic rhinitis, hay fever, urticaria, other allergic conditions, or any respiratory infection within two months, cardiac disease, chest deformity, or occupational exposure to hazardous substances. On the basis of nicotine dependent score ascertained by WHO recommended Fagerstrom Test for Nicotine Dependence questionnaire, only the subjects who were in the category of light (score $\leq 3$ ) and medium (score with 4 \& 5) smokers with the smoking history $\leq 5$ years were taken in the study group. A summary of the study group follows: i) current tobacco smokers with average ND score of 2.29 \pm 0.64 ; and ii) baseline pulmonary function values $>80 \%$ (FVC, FEV1, PEFR, FEF25-75\%).

All subjects signed a written informed-consent form approved by the BPKIHS and NGMC ethical committee. The subjects were instructed not to involve in any high physical activities, not to take tobacco, alcohol, cigarette smoke for at least 12 hours before coming to the experiment. The experiment was done in the morning hours from 8:00am to $12: 00 \mathrm{pm}$ to avoid any circadian variation in the pulmonary function and the subjects were advised to come two hours after the light breakfast.

\section{Subject preparation and recording baseline pulmonary function test}

The subjects were familiarized with air-conditioned set up maintained at temperature $(25 \pm 2 \circ \mathrm{C})$ and humidity (65.62 $\pm 7.79 \%)$ of the comfortable zone. After the instruction of whole procedure of experiment and supine rest of 15 minutes with physical and mental relaxation, the baseline recording of PFT started. PFT was performed following FVC maneuver with a closed nose and connected directly by a mouthpiece to digital pneumotachograph, Medspiror, made in India. Subjects with inadequate FVC maneuver performance were excluded during the experiment. To perform spiromtery, subjects inspired fully, and then exhaled forcefully into the mouthpiece as long as possible. The PFT indices analyzed were forced vital capacity(FVC), forced expiratory volume in one second(FEV1), forced expiratory flow at $25 \%$ of FVC(FEF25\%), forced expiratory flow at $50 \%$ of FVC (FEF50\%), forced expiratory flow at $75 \%$ of FVC (FEF75\%), and forced expiratory flow at middle half of FVC (FEF25-75\%).

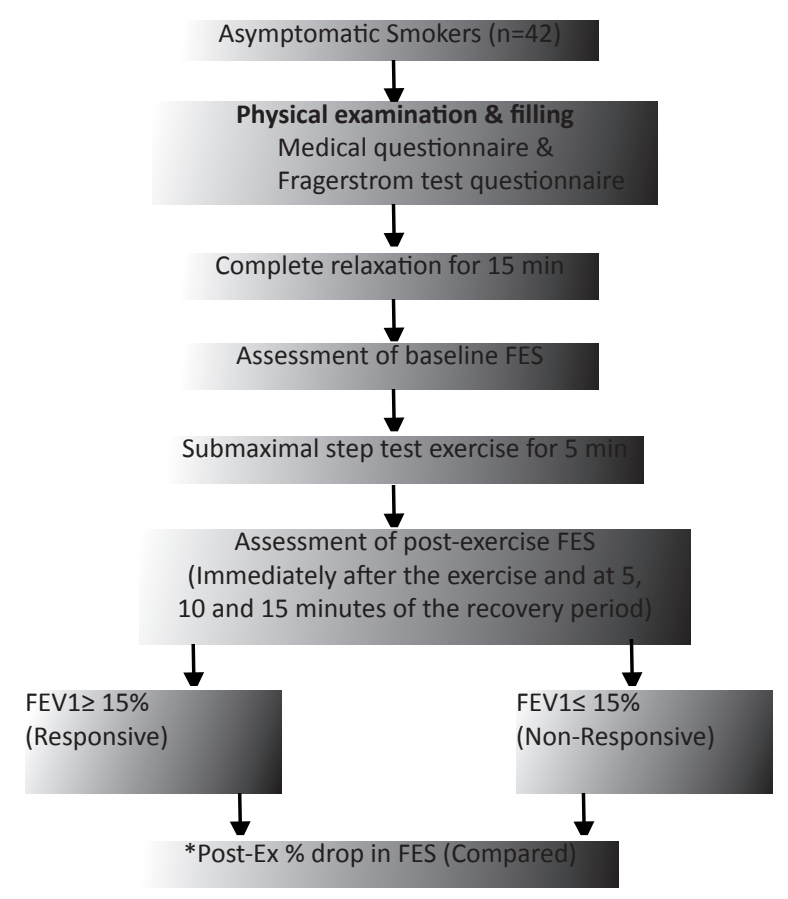

Figure 1. Experimental design.

\section{Exercise challenge testing and Recording of post-exercise PFT}

Standardized exercise challenge protocol based on the guideline of American thoracic society for methacholine and exercise challenge testing-1999 was followed. According to the protocol, performed step test exercise on 12 inches high bench to achieve 80 to $90 \%$ of the maximum predicted heart rate (220-age) for $5 \mathrm{~min}$. Immediately after the completion of the exercise challenge PFT was recorded according to standard protocol.8 The other three subsequent recordings were taken at 5, 10 and 15 minutes of recovery period after the challenge with $5 \mathrm{~min}$ interval of sitting rest. For the subject to be considered hyperresponsive to the exercise challenge, the greater 
than equal to $15 \%$ drop in FEV1 from the baseline value was required to be manifested in the recovery period.

\section{Calculation and statistical method employed}

Values are expressed as means and standard errors, except for Nicotine Dependence (ND) Score, age, height which are given as means and standard deviations. The significance difference between the two subgroups was analyzed using non-parametric tests such as Kruskul-Wallis and MannWhitney $U$ test and to compare difference of the means between baseline and post-exercise values among smokers Wilcoxon Signed Ranks test was used. The values for ' $p$ ' less than 0.05 were deemed significant. For all the statistical analyses, SPSS software was used.

Table 1. Comparison of demographic data and baseline FES of smokers with responsive and non-responsive to the exercise challenge.

\begin{tabular}{|lll|} 
& Responsive $(n=22)$ & Non-responsive $(n=20)$ \\
\hline Age(years) & $21.92 \pm 0.51$ & $22.33 \pm 0.77$ \\
\hline Height (cms) & $170.58 \pm 1.66$ & $168.78 \pm 2.80$ \\
\hline Weight (Kgs) & $62.67 \pm 2.20$ & $61.78 \pm 2.25$ \\
\hline ND scores & $2.25 \pm 0.19$ & $2.33 \pm 0.71$ \\
\hline FVC (Liters) & $3.07 \pm 0.13$ & $3.10 \pm 0.11$ \\
\hline FEV1 (Liters) & $2.83 \pm 0.12$ & $2.75 \pm 0.08$ \\
\hline FEV1/FVC\% & $90.1 \pm 1.71$ & $88.55 \pm 1.26$ \\
\hline FEF25-75\% (L/min) & $3.41 \pm 0.28$ & $3.04 \pm 0.17$ \\
\hline PEFR (L/min) & $8.16 \pm 0.43$ & $8.24 \pm 0.18$
\end{tabular}

Values are mean \pm SE; ND, Nicotine dependence scores; FEV1, forced expiratory volume in 1 second; FVC, forced vital capacity; FEF25-75\%, forced expiratory flow at middle half of FVC; PEFR, peak expiratory flow rate. There is no statistically significant different value between the two groups.

\section{RESULTS}

Volunteers participated in the study had no significant differences in age, height, weight, and ND scores (Table 1). Twenty-two of the forty-two subjects taken had a $\geq 15 \%$ drop in FEV1 at submaximal exercise challenge, and they were grouped under the category of responsive asymptomatic smokers and others of non-responsive asymptomatic smokers.

During exercise challenge maximum heart rate response recorded, did not vary among the subjects of both subgroups with an average HR of $85.96 \pm 7.05 \%$ of the maximum predicted heart rate (220 minus age in years).

\section{FEV1 as the measured variable}

The time pattern of changes indicating the response characteristics of the participants, the Mean \pm SE values for FEV1at the baseline and after the $5 \mathrm{~min}$ of exercise challenge at $0,5,10,15$ minutes of recovery period is shown in figure $\mathrm{A} 1$, for the both subgroups of smokers.
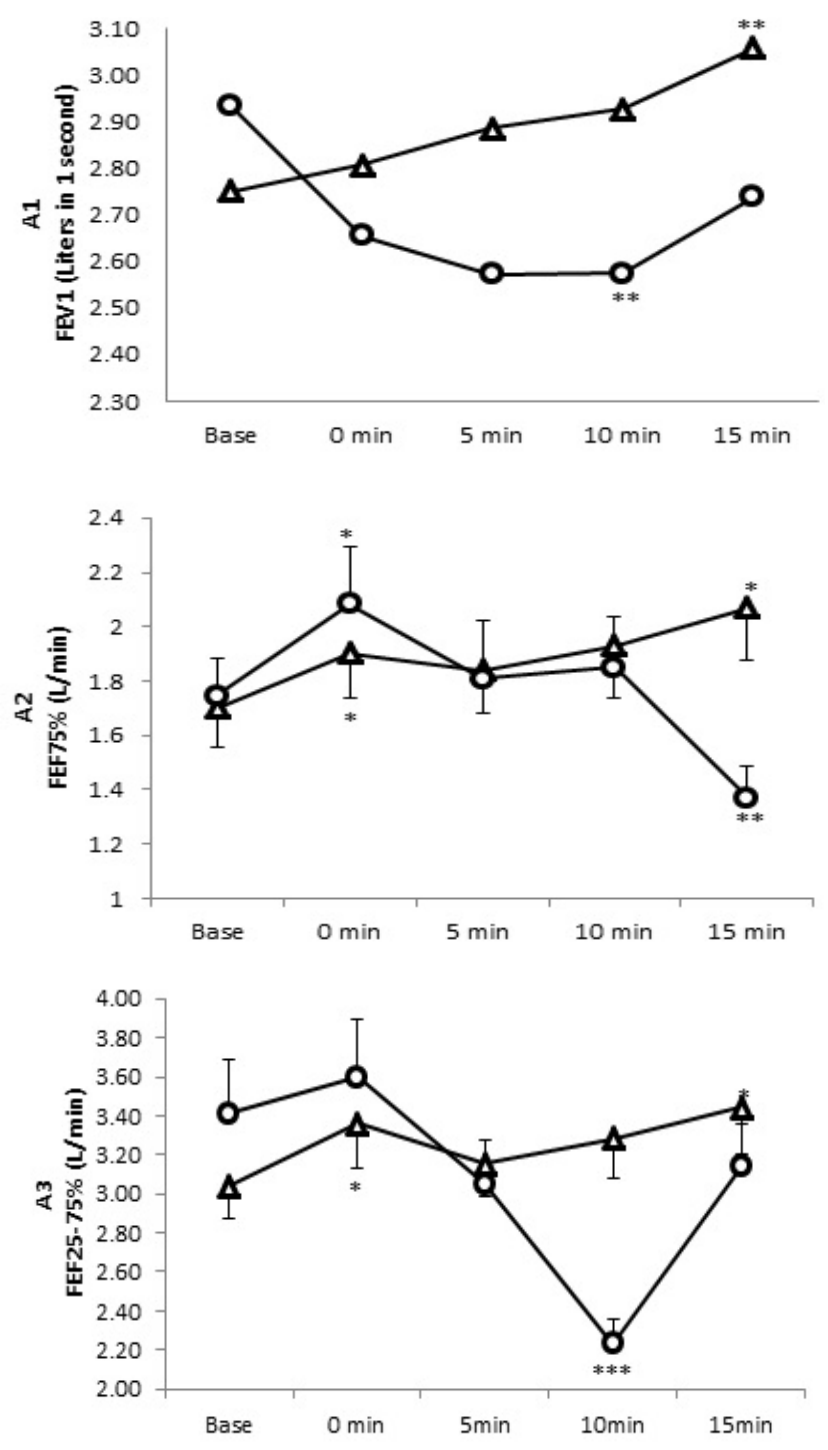

Figure 1. Time pattern changes in FEV1(A1), FEF75\%(A2) and FEF25-75\%(A3) respectively at $0,5,10$, and 15 minute from the baseline among Responsive (--O--) and Non-Responsive (--- $\Delta---)$ smokers.

$(*)=p$-value $<0.05,\left({ }^{* *}\right)=p$-value $<0.01$, and $\left({ }^{* * *}\right)=$ P-value $<0.001$ indicate the significant of difference from the baseline value in each group.

The values dropped from the baseline value $(2.93 \pm 0.12)$ among responsive smokers, which was significant $(p<0.05)$ at $0 \mathrm{~min}(2.65 \pm 0.11), 5$ minute $(2.57 \pm 0.12), 10$ minute $(2.57 \pm 0.12), 15$ minute $(2.74 \pm 0.13)$ of recovery period. In case of non-responsive smokers values increased from the baseline and found significant $(p<0.05)$ at 15 min of post exercise recovery period.

The percentage fall in FEV1 ( $\triangle \mathrm{FEV} 1 \%)$ was calculated from the baseline and maximum percentage fall was selected out of recovery period for each, which is shown in figure 2 . The average values of maximum $\%$ fall found were $21.23 \pm 1.26 \%$ and $2.75 \pm 2.56 \%$ at $p<0.001$ of significant difference for responsive and non-responsive subgroups of smokers respectively. Among the responsive smokers, six presented with maximal \% fall with FEV $1 \geq 15 \%$ at 0 


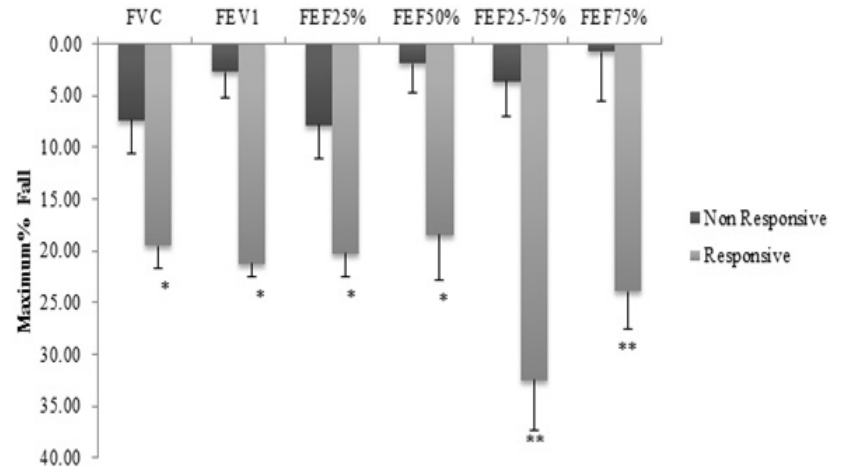

Figure 2. Maximum percentage fall in FES parameter in the post exercise state from the baseline values.

$(*)=p$-value $<0.01$, and $(* *)=p$-value $<0.001$ indicate the significant of difference between the Responsive and Non Responsive young adult asymptomatic smokers.

minute, 8 at 5 minutes and 8 at 10 minutes of post exercise recovery period.

\section{FEF75\% as the measured variable}

Recovery FEF75\% values at the time pattern of $0,5,10,15$ minutes as depicted in the figure $1(A 2)$, showed significantly increased $(p<0.01)$ from the baseline at 0 minute for the both subgroups of smokers. However, responsive smokers presented a trend of decline in the recovery period and found a significant drop $(1.37 \pm 0.4, p<0.01)$ from the baseline at 15 minute.

Mean FEF75\% was the maximum percentage fall in FEF75\% from the baseline as shown in figure 2 . In most of the responsive group (21 of 22 ) the fall was evident at 15 minute of the recovery period and one subject at 5 minute with average of $23.94 \pm 3.68 \%$. The non-responsive smokers showed considerably lower $(p<0.001)$ value of $0.82 \pm 4.72 \%$ than the responsive ones.

\section{FEF25-75\% as the measured variable}

The time pattern of changes in FEF25-75\% is shown in the figure $1(A 3)$. Although, responsive group had higher baseline FEF25-75\% values (Table 1.) than the non-responsive, the difference was insignificant. Post exercise recovery values $(2.23 \pm 0.13)$ dropped significantly $(p<0.001)$ at 10 minutes from the baseline and followed improvement at 15 minutes among responsive group. However, the values were higher among the non-responsive group and reached significant $(p<0.05)$ at 15 minutes of recovery.

FEF $25-75 \%$, the mean of maximum percentage fall in FEF25-75\% from its baseline is shown in figure B. Among the responsive group, most of them had maximum drop $(32.50 \pm 4.79 \%)$ with twenty participants at 10 minutes and two at 15 minutes of recovery. Majority of the nonresponsive smokers had very low $(p<0.001)$ post exercise maximum percentage drop (3.64 $\pm 3.32 \%)$ except one, which had $23.51 \%$ drop from the baseline at 10 minutes.

\section{FEF25\% as the measured variable}

No significant difference observed comparing baseline and time pattern values. However, the average of maximum $\%$ fall in FEF25\% (FEF25\%), shown in figure 1, was lower $(p<0.01)$ in responsive $(20.10 \pm 2.18 \%)$ than non-responsive group $(7.88 \pm 3.23 \%)$. Eight of the responsive smokers had maximum \% drop at 5 minutes, six at 15 minutes, four at 0 minute and four at 10 minutes of recovery period.

\section{FVC and PEFR as the measured variable}

Analysis of FVC time pattern showed the value $(2.73 \pm 0.12)$ at 10 minute dropped significantly $(p<0.05)$ from the baseline (table 1.) in the responsive group and improved at 15 minutes of recovery. However, the pattern in nonresponsive group indicated gradual increment, but remained insignificant. Mean of FVC fall differed (Responsive Vs Non-responsive: $19.51 \pm 2.21 \%$ Vs $7.43 \pm 3.15 \%)$ significantly $(p<0.01)$ between the groups.

The time pattern analysis of peak expiratory flow rate (PEFR) in responsive showed significant $(p<0.01)$ drop from the baseline at 5 minutes $(8.25 \pm 0.53$ Vs $7.02 \pm 0.29)$ and recovered at 15 minutes, whereas non-responsive had unaltered recovery PEFR pattern. The difference in the maximum percentage fall in PEFR (PEFR) was also found significant $(p<0.05)$ between the groups.

\section{DISCUSSION}

Through the assessment of the pulmonary function test following exercise challenge, the present study demonstrated that the responsive subset of smokers is associated with significantly decreased airway mechanics. Evaluating in term of a sensitive FES parameter FEV1, 22 asymptomatic smokers out of 42 taken who were found responsive had drop in FEV1 more than $15 \%$ within 15 minutes of post exercise recovery period. The analysis of the post exercise time pattern changes in other FES parameters (Figure 1 (A1, A2 and A3)) including FVC and PEFR in the responsive smokers indicated marked drop from the baseline at the different time points, except FEF $75 \%$ at 0 minute. It shows that bronchodilatation response to exercise is weak in these smokers which may be because of the associated heightened pathophysiology of inflammation of peripheral airways. ${ }^{9}$ A study in the asymptomatic smokers by Quaedvlieg et al also has demonstrated the bronchoconstrictive response to cold air challenge which is extending largely into the small peripheral airways by impedance measurement method which was not seen in normal subjects. ${ }^{10}$ However, they have not differentiated among the smokers with more sensitive and hyperresponsive to the modality of challenge used and there is no smokers sub categorization. Based on available studies till now for the first time Brown et al in 1977, exposed a group of asymptomatic smoker to inhale histamine showing responsiveness and demonstrated reactivity in the larger airways. ${ }^{11}$ In the current study, a clear trend of decline in FEV1 in responsive smokers with comparable history and nicotine dependence ascertained by Fagerstrom Test for Nicotine Dependence questionnaire, may emphasize that exercise challenge testing might 
be useful tool for screening mechanical abnormalities induced by smoke inhalation. Different processes might be responsible for the changes in airway function during and after in both modes of the challenges, but the post exercise decline in FEV1 was the pertinent fact in both of the cases.

There are ample evidences available which have shown a bronchoconstrictive response to various nonspecific stimuli including exercise challenge and airway hyper responsiveness to provocative agents in asymptomatic smokers having an alteration of ventilatory functions due to hyper reactive peripheral airways. ${ }^{12,13}$ The pathological changes found in smoker's lung which could be responsible for active muscle constriction and airway narrowing following the challenges may include: 1) airway epithelial damage, resulting in increased permeability and impairment of other epithelial function; 2) chronic airway inflammation; 3) structural changes in the airway wall; and 4) loss of alveolar attachments. However, one or more than one processes might be at work in smokers or might be not all smokers develop the abovementioned airway abnormalities. ${ }^{14}$

The effects of smoking on small or peripheral airways have been addressed by many studies in asymptomatic smokers. ${ }^{15-18}$ However, the effect of exercise challenge on peripheral airway mechanics in asymptomatic smokers have rarely been reported. As the peripheral airways is one of the determinant sites of ventilatory function in the human lung during physical activities and the first structure to be encountered by the smoke inhalation and earlier site of the mechanical deformity in smokers..$^{19,20}$ We conducted study in the asymptomatic smokers having no apparent differences between responsive and non-responsive subgroups on the basis of baseline values of FES before the intervention and other data related to age, sex, height, weight and ND scores (Table 1.). With sub-maximal exercise challenge testing of 5 minutes in the present study, the flow rates at lower volumes which primarily reflect the patency of small airway functions such as FEF 25\%, FEF25$75 \%$ and FEF75\% of vital capacity were found significantly different between the two groups of smokers in the recovery period. ${ }^{21,22}$ The observed post exercise maximum percentage fall in maximum mid expiratory flow rate (FEF25$75 \%$ ) was highest in case of responsive smokers (Figure B). But the fact of high variability of mean diameter of bronchioles the mean values of these parameters might be affected. ${ }^{23}$ However, FEF25-75\% is theoretically less effort dependent than FEV1 and is believe to be a measurement of medium sized peripheral airways. ${ }^{19}$ Even though guidelines of American thoracic society does not mention about the role of FEF25-75\% in the clinical assessment of airway resistance to flow, some of the studies has shown that $\mathrm{FEF} 25-75 \%$ is more sensitive indicator of airway resistance in symptomatic asthma than FEV1 in children and adults. ${ }^{24-29}$ In term of these parameters, current study suggested that the peripheral airway resistance response to exercise is more compromised among responsive asymptomatic smokers. The airway dilatation and thus decrease in airway resistance is a common physiological response to exercise for normal people, but it appears that partial airway obstruction and weak elastic recoil of lungs is evident in smokers which is leading to a subclinical alteration of ventilatory patterns of small or peripheral airways due to active cigarette smoking. ${ }^{30} \mathrm{~J}$ A Dosman et al in 1986, who evaluated peripheral airways in middle age heavy smokers by helium flow-volume curves and single breath nitrogen test, also indicates that the individuals who responded to inhaled histamine had increase in airways reactivity and peripheral airways dysfunction both, but not in non responsive smokers. ${ }^{30} 1$

Although, cigarette smoke causes the airway hyper responsiveness which is a primary risk factor for development of COPD (chronic obstructive pulmonary disease) or chronic bronchitis, most the smokers do not develop symptomatic COPD, probably because of the large physiological reserve of the lung. ${ }^{32}$ Interestingly, around 15$20 \%$ do experience accelerated loss of lung function, and the basis for heightened susceptibility in the smokers is yet to be known..$^{33}$ Observation of our study supports the point and suggest that there are distinctively two different groups of smokers; one responding to exercise challenge with maximum peripheral airway resistance due to underlying bronchial hyper responsiveness and another not. This leads to an interesting speculation that though there are smokers who are in the earlier phase of cigarette smoke induced mechanical deformity of airways, only few manifest airway hyper responsiveness to naturally occurring challenge such as exercise and could possibly predispose to clinically significant airway disease. ${ }^{30}$

\section{CONCLUSION}

Smoking causes alterations in lung health but may not necessarily manifest with development of disease or its manifestation by presentation of clinical symptoms. The present study has explored the extent and pattern of changes in peripheral airway resistance in young asymptomatic smokers and found no significant change in the basal values, however few of them exhibited altered airway response to exercise challenge, and that might identify among the smokers, the possible victim to develop obstructive airway disease in future. The observation of airways responses in our study is particularly based on a brief period of exercise challenge for 5 minutes which may be different in the different set ups of exercise and age and history of smoking which emphasize the need for further studies. The other limitation we deliberately came across was lack of consideration of passive smoke exposure duration among smokers which might also have influence on them. 


\section{REFERENCES}

1. Murshid KR. Asymptomatic gallstones: Should we operate? Saudi J Cosio Piqueras MG, and Cosio MG. Disease of airways in chronic obstructive pulmonary disease. Eur Respir J 2001;18:41S-49S.

2. Dan Stansescu, Antonio S, Claude Veriter, Annie R. Identification of smokers susceptible to development of chronic airflow limitation: A 13-yeas follow-up. Chest 1998; 144:416-25.

3. Betsuyaku T. The mechanism of airway obstruction in the development of COPD. Nippon Rinsho $2003 ; 61(12): 2133-7$.

4. Casale TB, Rhodes BJ, Donnelly AL, Weiler JM. Airway response to methacholine in asymptomatic nonatopic cigarette smokers. J Appl Physiol 1987 May; 62(5): 1888-92.

5. Martinez FD, Antognoni G, Macri F, Bonci E, Midulla F, De Castro G, Ronchetti R. Parental smoking enhances bronchial responsiveness in nine-year-old children. Am Rev Respir Dis 1988; 138:518-23.

6. Popovic-Grle S, Pavicic F, Bicanic V. The effect of cigarette smoking on pulmonary diffusing capacity in asymptomatic smokers. Lijec Vjen 1992; 114(5-8)118-21.

7. Andre S, Lucas M, Raposo M, Matos C, Abreu M. The effect of cigatette smoking on pulmonary diffusing capacity in asymptomatic smokers. Lijec Vjesn 1992; 114 (5-8):118-2.

8. The Official Statement of the American Thoracic Society. Guidelines for Methacholine and Exercise Challenge Testing-1999. Am J Respir Crit Care Med 2000; 161: 309-29.

9. Eggleston $\mathrm{PA}$, Rosenthal RR, Anderson $\mathrm{SD}$, Anderton R, Bierman CW, Bleecker ER, Chai H, Cropp GJ, Johnson JD, Konig P, Morse J, Smith $\mathrm{LJ}$, Summers RJ, and.Trautlein JJ. Guidelines for the methodology of exercise challenge testing of asthmatics. Allergy Clin Immunol1979; 64:642-45.

10. Quaedvlieg M, Wouters EF. In: Early airway obstruction in young asymptomatic smokers after cold air challenge. Respiration 1990, 57(5):299-303.

11. Brown NE, McFadden ER Jr. Ingrain RH Jr. Airway responses to inhaled histamine in asymptomatic smokers and nonsmokers. J Appi Physiol Respirat Environ Exer Physiol 1977; 42:508-13.

12. Finkelstein R, Ma HD, Ghezzo H, Whittaker K, Fraser RS, Cosio MG. Morphometry of small airways in smokers and its relationship to emphysema type and hyperresponsiveness. Am J Respir Crit Care Med 1995 Jul;152(1):267-76.

13. Teculescu DB, Pino J, Sadoul P. Cigarette smoking and density dependence of maximal expiratory flow in asymptomatic men. $\mathrm{Am}$ Rev Respir Dis 1980; 122(4):651-6.

14. Saetta M, Finkelstein R, Cosio MG.Morphological and cellular basis for airflow limitation in smokers. Eur Respir J 1994;7(8):1505-15.

15. Wright JL, Lawson LM, Pare PD, Wiggs BJ, Kennedy S, Hogg JC. Morphology of peripheral airways in current smokers and ex-smokers. Am Rev Respir Dis 1983; 127:474-77.

16. Walter S, Nancy NR, Collier CR. Change in the forced expiratory spirogram in young male smokers. Am Rev Respir Dis 1979; 119: 71724.
17. Amin K, Ekberg-Jansson A, Lofdahl CG, Venge P. Relationship between inflammatory cells and structural changes in the lungs of asymptomatic and never smokers: a biopsy study. Thorax 2003; 58(2):135-42.

18. Saetta M, Di Stefano A, Turato G, et al. CD8+ T-lymphocytes in the peripheral airways of smokers with chronic pulmonary disease. Am J Respir Crit Care Med 1998; 157:822-26.

19. Niewoehner DE, Knoke JD, Kleinerman J. Peripheral airways as a determinant of ventilatory function in the human lung. J Clin Invest 1977; 60:131-51.

20. McCormack DG, Salonen RO, Barnes PJ. Effect of sensory neuropeptides on canine bronchial and pulmonary vessels in vitro. Life Sci 1989; 45:2405-12.

21. Gelb AF, Zamel N. Simplified diagnosis of small-airway obstruction. N Engl J Med 1973;288:395-8.

22. Frank R, Liu MC, Spannhake EW, Mlynarek S, Macri K, Weinmann GG. Repetitive ozone exposure of young adults. Evidence of persistent airway dysfunction. Am J Respir Crit Care Med 2001;164:1253-60.

23. Cosio MG, Hale KA, Niewoehner DE. Morphologic and morphometric effects of prolonged cigarette smoking on the small airways. Am Rev Respir Dis 1980; 122:265-71.

24. Pellegrino R, Viegi G, Brusasco V, Crapo RO, Burgos F, Casaburi R, et al. Interpretative strategies for lung function tests. Eur Respir J 2005;26:948-68.

25. Miller MR, Hankinson J, Brusasco V, Burgos F, Casaburi R, Coates A, et al. Standardisation of spirometry. Eur Respir J 2005;26:319-38.

26. Lebecque $P$, Kiakulanda $P$, Coates AL. Spirometry in the asthmatic child: is FEF25-75 a more sensitive test than FEV1/FVC? Pediatr Pulmonol 1993;16:19-22.

27. Valetta EA, Piacentini GL, Del Col G, Boner AL. FEF25-75 as a marker of airway obstruction in asthmatic children during reduced mite exposure at high altitude. J Asthma 1997;34:127-31.

28. Lebowitz MD, Holberg CJ, Knudson RJ, Burrows B. Longitudinal study of pulmonary function development in childhood, adolescence, and early adulthood. Development of pulmonary function. Am Rev Respir Dis 1987;136:69-75.

29. Chiang C-H, Hsu K. Residual abnormalities of pulmonary function in asymptomatic young adult asthmatics with childhood-onset asthma. J Asthma 1997;34:15-21.

30. Buist AS,Vollmer WM, Johnson LR, Mccamant LE. Does the singlebreath N2 test identufy the smoker who will develop chronic airflow limitation? Am Rev Respir Dis 1988; 137:293-301.

31. Dosman J A, Bergstrom K, Clark K and Khaladkar S. Peripheral airways function and nonspecific airways reactivity in cigarette smokers. Chest $1986 ; 89: 45-8$.

32. Bates DV. The fate of chronic bronchitis: a report of 10 -years followup in the Canadian Department of Veteran's Affair coordinated study of chronic bronchitis. Am Rev Respir Dis 1973; 108:1043-65.

33. Betsuyaku T. The mechanism of airway obstruction in the development of COPD. Nippon Rinsho 2003 ;61(12):2133-7. 